\title{
Corpus
}

$10 \mid 2011$

Varia

\section{Des hypothèses, des tests et des données : les noms événementiels en corpus}

Delphine Beauseroy, Evelyne Jacquey et Marie Laurence Knittel

\section{(2) OpenEdition}

\section{Journals}

Édition électronique

URL : http://journals.openedition.org/corpus/2096

DOI : $10.4000 /$ corpus.2096

ISSN : $1765-3126$

Éditeur

Bases; corpus et langage - UMR 6039

Édition imprimée

Date de publication : 1 novembre 2011

Pagination : 219-238

ISSN : 1638-9808

\section{Référence électronique}

Delphine Beauseroy, Evelyne Jacquey et Marie Laurence Knittel, « Des hypothèses, des tests et des données : les noms événementiels en corpus », Corpus [En ligne], 10 | 2011, mis en ligne le 15 juin 2012, consulté le 07 septembre 2020. URL : http://journals.openedition.org/corpus/2096 ; DOI : https://doi.org/10.4000/corpus.2096 


\title{
Des hypothèses, des tests et des données : les noms événementiels en corpus
}

\author{
Delphine BEAUSEROY, Evelyne JACQUEY, \\ Marie Laurence KNITTEL \\ UMR 7118 ATILF, Nancy-Université
}

\section{Introduction}

L'objectif de notre travail est de confronter, sur la problématique des noms déverbaux, deux approches de la linguistique: l'approche introspective, fondée sur des tests et des hypothèses d'une part, et la linguistique de corpus, avec l'extraction, le décompte et l'analyse d'occurrences d'autre part.

Notre problématique est celle de la variété des lectures qu'admettent les noms déverbaux, et des propriétés morphosyntaxiques qui y sont liées. A des occurrences relevées dans un corpus selon un certain nombre de patrons morphosyntaxiques, nous avons appliqué une série de tests, élaborés à partir d'hypothèses sous-jacentes existant dans les travaux en linguistique introspective. Par le truchement des occurrences relevées dans le corpus d'étude selon les patrons syntaxiques choisis et satisfaisant les tests linguistiques utilisés, les hypothèses linguistiques seront confrontées avec les usages observés.

\section{La problématique des noms déverbaux : syntaxe et interprétation}

Dans le domaine de la syntaxe, notamment en grammaire générative, de nombreux travaux sur les noms déverbaux sont fondés sur l'hypothèse de Grimshaw (1990). Pour cet auteur, les noms déverbaux événementiels se subdivisent en deux catégories : les noms événementiels «simples» (NES) et les noms événementiels « complexes » (NEC), seuls susceptibles d'avoir des arguments. Selon Grimshaw, ces deux types fondamentaux 
D. Beauseroy, E. JACQuey, M. L. KNITTEL

se distinguent par leurs propriétés syntaxiques, résumées dans le tableau (1) :

Tableau 1. Propriétés syntaxiques des noms déverbaux événementiels (Grimshaw 1990)

\begin{tabular}{|l|l|l|}
\hline PROPRIÉTÉS & NEC & NES \\
\hline PROPRIÉTÉS & $\begin{array}{l}\text { structure } \\
\text { aspectuelle ; } \\
\text { structure } \\
\text { argumentale }\end{array}$ & $\begin{array}{l}\text { structure } \\
\text { aspectuelle }\end{array}$ \\
\hline $\begin{array}{l}\text { (i) ARTICLE EN 1 1 } \\
\text { MENTION }\end{array}$ & défini & variable \\
\hline $\begin{array}{l}\text { (ii) NOMBRE } \\
\text { invariable } \\
\text { (sing) }\end{array}$ & $\begin{array}{l}\text { variable } \\
\text { (sing/plur) }\end{array}$ \\
\hline \begin{tabular}{l} 
V CORRESPONDANT \\
\hline
\end{tabular} & obligatoire & facultatif \\
\hline
\end{tabular}

Grimshaw observe cependant des ambiguïtés entre noms événementiels et noms "résultatifs » (NR), c'est-à-dire renvoyant aux résultats des procès décrits par les verbes :

(1) a. La construction de la maison (a duré deux ans). [NEC]

b. La construction de mon voisin (défigure le paysage). [NR]

Par ailleurs, elle admet que les NEC peuvent adopter le fonctionnement des NES par une procédure de changement de type ('type shifting') :

(2) a. Les éleveurs ont filmé l'attaque du troupeau par les loups. [NEC]

b. Les éleveurs ont filmé \{une/plusieurs\} attaque(s) du troupeau. [NES]

Cette approche a été contestée par Knittel $(2008,2010 a)$ qui suggère au contraire que l'opposition entre NEC et NES cidessus ne représente en fait que des emplois différents d'une même unité lexicale, et non des classes distinctes. Plus précisément, les hypothèses de Knittel sont les suivantes :

(i) Les NEC se caractérisent par leur caractère massif; les NES et les noms résultatifs sont au contraire des noms comptables 
(Beauseroy \& Knittel 2007, Heyd \& Knittel 2009) ; l'opération de «type shifting» suggérée par Grimshaw consiste simplement à partitionner les référents décrits par ces noms (Kwon \& Zribi-Hertz 2004, Borer 2005).

(ii) $\mathrm{Du}$ fait de leurs propriétés sémantiques, seuls les $\mathrm{NE}$ construits sur des «once-only prédicates 》 (i.e. décrivant des événements ne pouvant affecter qu'une seule fois leur patient, e.g. construction, destruction, cf. de Swart 1991) résistent tant à la lecture pluriactionnelle, i.e. itérative (Van Geenhoven 2004, Laca 2006), qu'à la pluralisation (Knittel 2010b).

(iii) Les propriétés syntaxiques prêtées par Grimshaw aux NEC (complément obligatoire, article défini) résultent de l'emploi de ces noms en construction possessive (Milner 1982, Godard 1986, Zribi-Hertz 1998, Alexiadou 2001, Knittel 2010a), notamment s'ils sont apparentés à des verbes transitifs directs. Dans cet emploi, le NE doit présenter un complément déterminé, sauf si ce dernier est pluriel. La construction possessive n'est pas requise lorsque le complément est non quantifiable et indéterminé, indépendamment de son nombre. En conséquence, l'article défini ne s'impose pas dans ces contextes.

En somme, notre hypothèse est que les effets sémantiques, syntaxiques et morphosyntaxiques observés dans le champ des nominalisations sont le reflet de certains facteurs sous-jacents (e.g. opposition massif/ comptable; construction possessive ; itération (im)possible), et ne sont pas propres aux nominalisations. Notre objectif est donc de valider par une étude sur corpus les hypothèses ci-dessus, émises à partir d'exemples construits.

\section{Méthodologie de la recherche}

\section{1 Élaboration du corpus d'étude}

\subsubsection{Matériaux}

Notre objectif étant d'étudier les occurrences des noms déverbaux du français, nous nous sommes appuyées sur deux éléments :

- une liste de référence de ce type de noms : le lexique Verbaction de 9397 noms, élaboré sous la direction de N. Hathout à l'InalF 
puis à l'ERSS (2002), accessible en ligne et fondé sur la nomenclature du TLF (verbes et substantifs);

- un corpus de données journalistiques en français, à savoir trois années $(1999,2002,2003)$ du quotidien régional L'Est Républicain, mis à disposition par le journal auprès du serveur du centre de ressources le CNRTL.

3.1.2. Logiciel d'interrogation des données source: le concordancier Corpus Workbench

Notre choix s'est porté sur le concordancier Corpus Workbench (CWB, http://cwb.sourceforge.net/demos.php) car parmi les logiciels libres de ce type, il est le seul capable de supporter la quantité de données interrogées pour extraire les occurrences de noms déverbaux, à savoir environ 240 millions de mots. Ce logiciel supporte de telles masses de données parce que cellesci, une fois annotées en morphosyntaxe, sont mémorisées sous une forme indexée. D'autre part, ce logiciel a été utilisé dans des projets de grande envergure en termes de linguistique de corpus et d'analyse de la langue, en l'occurrence irlandaise, à visée lexicographique (Kilgariff \& Dhonnchadha 2006).

Pour extraire les occurrences de noms déverbaux, nous avons utilisé l'interface de CWB dans sa version minimale, reposant sur l'utilisation de lignes de commandes et d'un langage d'interrogation accessible pour des linguistes «CQP Syntax ». Dans la mesure où les données textuelles interrogées ont été annotées en morphosyntaxe et où ces annotations ont été indexées au même titre que les données brutes, les requêtes lancées au concordancier tiennent compte classiquement de l'ordre linéaire des unités recherchées et peuvent être enrichies de conditions sur les formes lemmatisées et les parties du discours. Par exemple, la requête ci-dessous permet d'interroger l'ensemble des données pour rechercher les occurrences du déverbal construction, précédé d'un déterminant défini et suivi d'un groupe prépositionnel en de comprenant un groupe nominal déterminé.

(3) $>[$ lemma $=$ 'le' $][$ lemma $=$ 'construction' \& pos='NOM'] $([$ lemma $=$ 'de] [pos='DET.*'] | [lemma $=$ 'du']) [pos='NOM'] ;

Outre le type de données interrogeables, l'interface de CWB permet de sauvegarder le résultat d'une requête, d'extraire une 
sous-partie des résultats - soit sur la base d'une quantité maximum décidée par l'utilisateur, soit par extraction aléatoire - et enfin de sauvegarder les résultats choisis sous la forme d'un fichier texte tabulaire.

\subsubsection{Extraction des concordances étudiées}

Munis des données textuelles source, du concordancier et de son langage d'interrogation, nous avons été en mesure de rechercher les quatre patrons distributionnels ci-dessous, correspondant à des configurations morphosyntaxiques associées aux hypothèses que nous voulions confronter aux usages des noms débervaux. Ces quatre patrons font varier la détermination du nom déverbal (définie $[1,4]$ ou indéfinie $[2,3]$ ) et celle du groupe prépositionnel qui lui est directement consécutif (présence d'une détermination $[1,2]$ ou non $[3,4]$ ).

Tableau 2. Patrons distributionnels recherchés

\begin{tabular}{|l|l|l|l|}
\hline & ARTICLE & & COMPLÉMENT \\
\hline 1 & défini & (Adj) NE (Adj) & $\begin{array}{l}\text { déterminé / indéfini } \\
\text { pluriel (Adj) }\end{array}$ \\
\hline 2 & indéfini & (Adj) NE (Adj) & déterminé (Adj) \\
\hline 3 & indéfini & (Adj) NE (Adj) & indéterminé (Adj) \\
\hline 4 & défini & (Adj) NE (Adj) & indéterminé \\
\hline
\end{tabular}

Le tableau (3) ci-dessous synthétise les résultats obtenus, pour chaque patron distributionnel et pour l'ensemble des noms listés dans le lexique Verbaction.

Tableau 3. Quantité de résultats par patron distributionnel

\begin{tabular}{|l|r|r|r|}
\hline & $\begin{array}{c}\text { NE non modifié } \\
\text { par un adjectif }\end{array}$ & $\begin{array}{c}\text { NE modifié par } \\
\text { un adjectif }\end{array}$ & \multicolumn{1}{|c|}{ TOTAL } \\
\hline 1 & 451002 & 48822 & 499824 \\
\hline 2 & 42755 & 23832 & 66587 \\
\hline 3 & 101610 & 19706 & 121316 \\
\hline 4 & 41348 & 8492 & 49840 \\
\hline
\end{tabular}

$\mathrm{Au}$ vu de la quantité de données à analyser pour le premier patron, nous avons procédé à deux sélections. Premièrement, une liste réduite de 10 déverbaux, appartenant à des 
D. Beauseroy, E. Jacquey, M. L. KNitTel

classes aspectuelles différentes et construits au moyen de suffixes divers, a été extraite de Verbaction: acheminement, aménagement, blocage, construction, création, évolution, ouverture, nettoyage, rejet, remontée. Deuxièmement, nous avons arrêté le nombre d'occurrences à analyser manuellement à 200 par annotateur pour des raisons de faisabilité. La limitation à 200 résultats a été opérée à l'aide de la fonctionnalité d'extraction aléatoire fournie dans CWB. Pour le premier patron, les 200 occurrences correspondent à 20 occurrences pour chacun des 10 déverbaux choisis et tiennent compte des proportions d'occurrences au singulier et au pluriel.

Pour les trois autres patrons distributionnels, la restriction initiale à dix déverbaux risquait de produire un nombre de résultats trop faible par rapport à ceux du premier patron. Par conséquent, nous n'avons gardé que la seconde restriction limiter le nombre de résultats à analyser manuellement à 200 par annotateur - et en appliquant la proportion de 150 occurrences au singulier et 50 au pluriel.

A l'issue de cette étape d'extraction, nous obtenons donc un «corpus d'étude » constitué de 200 résultats avec leurs contextes gauche et droit, pour chaque patron distributionnel et pour chacun des deux annotateurs. Au final, le corpus d'étude sur lequel est bâti le travail présenté est donc constitué de " concordances », c'est-à-dire de 1600 occurrences de noms déverbaux en contexte journalistique qui sont ensuite analysées manuellement.

\subsection{Analyses des concordances extraites}

\subsubsection{Distinction NE/NR}

Rappelons en premier lieu que, selon Grimshaw (1990), mais voir également Melloni (2007), certains noms déverbaux sont ambigüs entre une lecture événementielle et une lecture résultative (cf. exemple (2)). Dans la mesure où notre étude porte seulement sur les noms en lecture événementielle, il est nécessaire de détecter et d'isoler les noms à interprétation résultative du corpus d'étude. Dans un premier temps, les 1600 occurrences extraites ont donc été testées pour déterminer le caractère [ \pm événementiel] du nom-tête. Pour ce faire, deux principales catégories de tests ont été mises en place : des tests 
aspectuels (issus du projet ANR Nomage, Haas, Huyghe et Marín 2008) et des tests sur la structure argumentale (élaborés à partir des observations de Grimshaw 1990); à ces deux ensembles ont été ajoutés d'autres tests proposés par Grimshaw, des tests portant strictement sur la lecture résultative, ainsi qu'un test portant sur la pluralisation (voir section 2, (iii)). L'ensemble des tests est fourni en annexe. N'ont été retenus que les noms répondant positivement aux tests aspectuels et argumentaux, et négativement aux tests résultatifs.

\subsubsection{Confrontation aux hypothèses}

Après avoir isolé les occurrences de noms dénotant des résultats, c'est-à-dire répondant négativement aux tests aspectuels et argumentaux, nous avons confronté les critères de classification de Grimshaw aux occurrences extraites pour chaque patron, afin d'en vérifier la pertinence. Outre les propriétés aspectuelles (NES et NEC) et argumentales (NEC) qu'elle associe aux NE, Grimshaw fait l'hypothèse que ces classes, qu'elles soient d'occurrences ou d'unités lexicales, sont caractérisables à l'aide de propriétés morphosyntaxiques concernant la détermination du nom événementiel et sa pluralisation (cf. Tableau 1). L'ensemble des propriétés réunies par Grimshaw permet ainsi de subdiviser l'ensemble des occurrences extraites du corpus au travers de 8 classes. Les résultats sont fournis dans le tableau (4) ; les lignes grisées correspondent aux structures impossibles selon Grimshaw :

Tableau 4. Nombre d'occurrences des noms événementiels selon les patrons morphosyntaxiques

\begin{tabular}{|c|c|l|l|c|}
\hline & ARGUMENTS & $\begin{array}{l}\text { ARTICLE EN } \\
\text { TÊTE DE } \\
\text { STRUCTURE }\end{array}$ & $\begin{array}{l}\text { NOMBRE } \\
\text { DE NE }\end{array}$ & $\begin{array}{l}\text { NOMBRE } \\
\text { D’OCCURRENCES } \\
\text { RELEVÉES }\end{array}$ \\
\hline 1 & + & Défini & Singulier & 174 \\
\hline 2 & + & Défini & Pluriel & 22 \\
\hline 3 & + & Indéfini & Singulier & 66 \\
\hline 4 & + & Indéfini & Pluriel & 5 \\
\hline 5 & - & Défini & Singulier & 73 \\
\hline 6 & - & Défini & Pluriel & 21 \\
\hline 7 & - & Indéfini & Singulier & 137 \\
\hline 8 & - & Indéfini & Pluriel & 12 \\
\hline
\end{tabular}


D. Beauseroy, E. Jacquey, M. L. KNitTel

\section{Résultats}

Confrontées aux hypothèses avancées dans la section 2, les données présentées dans le tableau (4) ci-dessus permettent de formuler un certain nombre de conclusions, qui, selon les cas, valident ou invalident les hypothèses de départ.

\subsection{Examen des hypothèses de départ}

\subsubsection{Propriétés des noms événementiels complexes}

Rappelons en premier lieu que, selon Grimshaw, les noms événementiels apparaissent toujours au singulier et précédés de l'article défini, soit selon le type 1 du tableau 4 ci-dessus (cf. exemple (6)).

Or, notre étude de corpus indique que des noms événementiels associés à leurs arguments, et donc complexes au sens de Grimshaw, apparaissent aussi au pluriel et/ou introduits par des articles indéfinis. Le type 1 du tableau 4, considéré par Grimshaw comme étant le seul possible (6), ne représente en fait qu'une tendance majoritaire. La présence d'arguments n'est donc incompatible ni avec la pluralisation d'un NE (7), ni avec son introduction par l'article indéfini (8), ni avec les deux phénomènes conjoints (9).

(4) Il a également la charge de mener à bien l'acheminement $d u$ courrier dans plus de 3000 foyers. [31 août 1999, Pages spécifiques de Besançon, «Homme d'écoute »]

(5) J'observe la stratégie, les replacements, les remontées $d u$ ballon, la manière d'exercer le pressing. [19 janvier 2003, Pages spécifiques Sports Lorraine, « Nancy va s'en sortir »]

(6) La CAF pourra en cas de besoin procéder à un contrôle de leur situation. [21 août 1999, Pages spécifiques de Belfort, « Déclaration de situation »]

(7) A noter également, la participation de compétiteurs dans le domaine halieutique, qui ont proposé des démonstrations de différentes techniques de pêche. [6 septembre 1999, Pages spécifiques de Belfort, «Histoire du permis de pêche »]

L'étude de données attestées nous a donc permis d'invalider l'hypothèse émise par Grimshaw quant aux propriétés des noms événementiels complexes, puisqu'on rencontre effectivement 
des configurations distributionnelles qui ne sont pas censées exister, même si elles sont moins fréquentes.

\subsubsection{Pluralisation}

La présence de noms événementiels au pluriel dont il vient d'être question nous a amenées à nous interroger plus avant sur la distribution du pluriel et les facteurs qui la conditionnent. En l'occurrence, l'étude de notre corpus a permis de mettre en évidence trois phénomènes.

D'une part, et comme nous le supposions, les noms renvoyant à des événements qui ne peuvent s'appliquer qu'une fois à un patient donné n'apparaissent pas au pluriel (cf. section 2 (ii)), et ce, même lorsqu'ils renvoient à plusieurs procès affectant chacun un patient particulier. L'exemple (8b) illustre ce cas de figure, l'exemple construit (8c) venant ici en contraste.

a. Ces terrains seront consacrés à la création d'un hôtel. [22 mai 1999, Pages spécifiques à l'édition d'Epinal, « Inova 3000 : extension »]

b. La rainette gonflable et la fabrication de cerfs-volants font partie des autres animations gratuites de l'autoroute. [6 juillet 1999, Pages spécifiques à l'édition de Neufchâteau, «Le jaune de la sécurité »]

[c. La rainette gonflable et les fabrications de cerfs-volants font partie des autres animations gratuites de l'autoroute.]

D'autre part, nous avons pu constater que les noms susceptibles d'être pluralisés admettent d'être employés au singulier alors même qu'ils réfèrent à une pluralité d'événements - ceci faisant écho à la remarque sur la non pluralisation des noms d'événements de Grimshaw, voir tableau 1. C'est ce qu'indique l'opposition entre (9a) et $(9 b)$ :

(9) a. Depuis un certain temps, les ravalements de façades s'accélèrent. [20 septembre 1999, Pages spécifiques à l'édition de Remiremont, « Les goûts et les couleurs »]

b. Des professionnels sont actuellement dans le secteur du Grand-Couronné pour effectuer le nettoyage des cheminées avant l'hiver. [1 $1^{\text {er }}$ août 1999, Pages spécifiques à l'édition de la Grande Banlieue Nord, «I.L.G.C de l'Amezule »] 
D. Beauseroy, E. JACQuey, M. L. KNITTEL

La présence d'un tel contraste a une valeur importante, puisqu'elle confirme l'une des observations faites par Knittel (2010a) (voir section 2, (ii)) : alors que l'exemple (9b) présente nettoyage des cheminées comme un événement unique, puisque singulier, constitué de plusieurs sous-événements de «nettoyage d'une cheminée », l'exemple (9a) présente chaque «ravalement d'(une) façade " comme un événement indépendant en soi, le pluriel signalant l'existence de plusieurs événements de nature identique. En d'autres termes, le référent de nettoyage (9b) a un fonctionnement cumulatif (i.e. il est non-pluriel alors même qu'il est constitué de multiples sous-événements), alors que celui de ravalements en (9a) est conçu comme une série d'événements portant chacun sur un patient particulier, d'où la présence du pluriel. On observe en outre que ce contraste est lié à la (non-)détermination du complément, un fait qui, jusqu'alors, n'avait été perçu comme pertinent que vis-à-vis de la distribution de la construction possessive (voir section 2, (iii)).

Enfin, nous avons constaté qu'il existe une autre classe de noms qui résistent à la pluralisation, comme le montrent les exemples qui suivent :

(10) Il autoriserait la desserte des villes moyennes par l'utilisation de demi-rames de TGV. [22 juillet 1999, Pages spécifiques à la Région Lorraine, pas de titre donné, article au sujet du TGV Est]

(11) Elle comporte un vide sanitaire qui n'a pas peu contribué à la préservation de remarquables fresques [...]. [25 juin 1999, Pages spécifiques à l'édition d'Epinal, « Adam en Eden »]

Outre le caractère non-itérable du prédicat nominal, Knittel (2010b) suggère qu'un autre facteur empêchant la pluralisation réside dans le fait que l'expression nominale renvoie à une activité pragmatiquement régulière. Toutefois, si ce facteur peut être évoqué pour rendre compte de (10), il ne semble pas en cause en (11). Au contraire, il semble que ce phénomène trouve son origine dans le type aspectuel de l'expression, le pluriel étant difficilement compatible avec les Activités. Or, l'hypothèse de l'incompatibilité du pluriel avec les expressions dénotant des événements non-bornés a été formulée par Mourelatos (1978) et par Borer (2005). Dans ce cas précis, on peut donc 
observer que l'étude du corpus nous conduit à rejeter l'une des hypothèses de départ au profit d'une autre qui n'avait pas été envisagée au moment de l'étude.

\subsubsection{Distribution des déterminants}

Du côté de la distribution des déterminants, nous avons pu constater, grâce aux concordances analysées manuellement, que la distribution des articles indéfinis ne se limite pas aux cas où le complément est non-quantifiable et indeterminé, comme en (12), ce qui constituait l'une des hypothèses de Grimshaw dont nous sommes parties :

(12) a. Cette intervention pourra entraîner une baisse de pression, voire un manque d'eau pour les habitants. [30 juin 1999, Pages spécifiques à l'édition de Lunéville, «A l'écoute de la mairie »]

b. A noter également, la participation de compétiteurs dans le domaine halieutique, qui ont proposé des démonstrations de différentes techniques de pêche. [6 septembre 1999, Pages spécifiques de Belfort, «Histoire du permis de pêche »]

En effet, plusieurs autres types de structures introduites par des déterminants indéfinis ont pu être repérés dans le corpus d'étude extrait à partir de l'Est Républicain.

Ainsi, dans les deux exemples qui suivent, on constate la conjonction de deux facteurs distinctifs : le fait que l'expression nominale se trouve dans un contexte hypothétique, dû à l'emploi $\mathrm{du}$ futur en (13) et de risques en (14), et le caractère sémantiquement indéterminé de prix en (13) et de partie en (14) ${ }^{1}$ :

(13) a. Les producteurs de fruits et légumes se réuniront pour discuter des modalités d'un double affichage des prix. [18 août 1999, Pages spécifiques informations générales, pas de titre particulier]

à comparer à l'exemple construit :

b. Les producteurs de fruits et légumes se réuniront pour discuter du double affichage des prix.

(14) a. Il explique les risques d'un effondrement d'une partie de la digue. [2 janvier 2002, Pages spécifiques à l'édition de la Meuse, «Quatre familles évacuées à Belleville»]

1 Nous remercions le relecteur de Corpus d'avoir signalé cette possibilité. 
D. Beauseroy, E. JACQuey, M. L. KNITTEL

à comparer à l'exemple construit :

b. Il explique les causes de l'effondrement d'une partie de la digue.

D'autre part, nous avons aussi constaté que la présence d'un adjectif modifiant le nom événementiel semblait un facteur favorisant l'emploi de l'article indéfini :

(15) a. ...un chemin piétonnier entre les [deux] villages [...] afin d'assurer une meilleure protection des marcheurs $[19$ janvier 2002, Pages spécifiques à l'édition de Bar le Duc, «Trois nouvelles associations »]

à comparer aux exemples construits :

b. afin d'assurer la protection des marcheurs

c. ?? afin d'assurer une protection des marcheurs

(16) a. La rue Gohypré bénéficiera d'un enfouissement complet de ses réseaux. [17 septembre 1999, Pages spécifiques à l'édition d'Epinal, «Programme des travaux »]

à comparer aux exemples construits :

b. La rue Gohypré bénéficiera de l'enfouissement de ses réseaux.

c. La rue Gohypré bénéficiera d'un enfouissement de ses réseaux.

De ce point de vue, les noms événementiels ne semblent pas fonctionner différemment de certains massifs concrets ou des noms désadjectivaux, pour lesquels la modification adjectivale entraîne le remplacement de l'article partitif par l'indéfini singulier (e.g. montrer \{de la gentillesse/une gentillesse exceptionnelle\}, voir Kleiber 2003, Kupferman 2004 pour les concrets, ainsi que van de Velde 1995, Beauseroy 2009 pour les abstraits désadjectivaux).

Les exemples qui précèdent révèlent donc l'un des apports de l'étude de corpus: elle permet de repérer des configurations distributionnelles qui n'étaient pas prévues dans les hypothèses de travail initiales. C'est donc bien la prise en compte de nouveaux contextes (contexte hypothétique, complément sémantiquement indéterminé, présence d'adjectifs), qui n'avaient pas été envisagés au départ, mais se sont imposés à 
partir du corpus lui-même, qui met en lumière de nouveaux phénomènes, et, par le biais de leur étude, incite à formuler de nouvelles hypothèses descriptives.

Cependant, les exemples qui suivent indiquent que les trois hypothèses mentionnées plus haut sont insuffisantes pour rendre compte de la distribution des indéfinis :

a. [...] qui menèrent à une étude de l'évolution de la calligraphie et des outils scripteurs [23 mai 1999, Pages spécifiques à l'édition d'Epinal, «A la découverte de l'héraldique »]

b. la journée s'est terminée par une visite du village alsacien [15 juin 1999, Pages spécifiques à l'édition de la grande banlieue Nord, « Escapade à l'écomusée d'Alsace »]

c. [II] avait été victime d'une fracture de la clavicule. $\left[1^{\mathrm{er}}\right.$ septembre 1999, Pages spécifiques sport national, « Jouer un rôle de premier plan », article au sujet du coureur Jalabert]

Actuellement, de tels exemples ne peuvent recevoir une analyse satisfaisante. Il est sans doute nécessaire de collecter plus de structures de ce type, et d'en réaliser une étude systématique afin de parvenir à caractériser leurs propriétés.

\subsection{Perspectives}

L'un des principaux intérêts de notre étude de corpus réside dans le fait qu'elle a permis de mettre au jour des données inattendues, qui débouchent sur de nouvelles perspectives de recherche. Ainsi, nous avons été confrontées à une série de noms événementiels intrinsèquement pluriels (18), série qui n'a, à notre connaissance, pas été décrite dans la littérature :

(18) a. Devant les applaudissements de nombreux spectateurs, parents et amis, ces poussins ont démontré toutes leurs techniques. [27 mai 1999, Pages spécifiques à l'édition de Remiremont, « Basket : victoire des poussins »]

b. Tout le monde regarde vers octobre 2003, date du début des travaux du pôle santé. [9 janvier 2002, Pages spécifiques à l'édition de Bar le Duc, «Les ambitions futures »]

c. Sur la piste, les évolutions langoureuses d'une splendide danse orientale ont fait monter la température de plusieurs degrés. [3 janvier 2002, Pages spécifiques à l'édition de Besançon, « Coup de chaleur sur la Saint-Sylvestre »] 
D. Beauseroy, E. JACQuey, M. L. KNITTEL

Un autre cas dans lequel les données se sont révélées inattendues est celui de la distribution des déterminants indéfinis, notamment dans le cas présenté sous (17), qui amènera peutêtre à une remise en cause des analyses classiques de la distribution des articles introduisant les noms événementiels.

\section{Conclusion}

Comme les données qui précèdent l'ont montré, le travail sur des occurrences relevées en corpus nous a non seulement permis de valider certaines hypothèses de départ, mais a également soulevé de nouveaux questionnements, à propos desquels de nouvelles hypothèses devront voir le jour.

Toutefois, avant de clore cet article, il convient de revenir sur les buts que nous nous étions fixés au départ, afin de les confronter à nos résultats. Le tableau (4) ci-dessus fait en effet mention de huit structures retenues au départ, dont la moitié seulement a pu faire l'objet d'une étude approfondie. Nous avons en effet rapidement constaté, pour les noms employés sans argument, que les facteurs discriminants quant à la détermination étaient à rechercher dans un contexte beaucoup plus large que celui qui avait été sélectionné (15 mots avant et 15 mots après). Autrement dit, la manière dont nous avons construit notre corpus ne nous a pas permis de dégager des facteurs reposant sur des unités de l'ordre du paragraphe.

Un autre problème auquel nous avons été confrontées concerne l'application des tests. Pour des raisons tenant, par exemple, à la nature ou à la complexité de la structure syntaxique dans laquelle se trouvait le nom à étudier, les tests se sont parfois révélés difficilement applicables sur l'exemple tel qu'il se présentait en corpus. La solution retenue a été, dans ce cas, de sélectionner le NE avec son complément, et de lui appliquer les tests sans tenir compte du contexte.

Dans le même ordre d'idée, l'utilisation d'exemples issus d'un corpus oblige à traiter toutes sortes de structures, dont certaines sont totalement ambigües, ou réagissent aux tests de manière erratique. Ainsi, en (19), remontées peut être interprété tant comme «le fait de remonter» (interprétation événementielle) que comme "ce qui remonte» (interprétation 
résultative); en (20), on constate que ouvertures se combine avec avoir lieu ce qui révèle son caractère événementiel, alors même qu'il renvoie aux périodes d'ouverture, autrement dit à ce qui résulte de l'action d'ouvrir.

(19) Le bâtiment sera construit sur vide sanitaire, pour éviter les remontées de la nappe phréatique. [29 août 2002, Pages spécifiques à l'édition de Vesoul, «Un coût de 5970000 euros »]

(20) Les prochaines ouvertures du secrétariat de la mairie auront lieu les samedis 26 juin et 3 juillet de 9 heure [...]. [24 juin 1999, Pages spécifiques à l'édition d'Epinal, «Ouverture de la mairie »]

Ces difficultés sont bien sûr absentes si l'analyse est réalisée à partir d'exemples construits, que l'on peut soigneusement élaborer pour la démonstration. Face à ce problème, nous avons fait le choix d'éliminer tous les exemples soulevant ce type de difficulté, car il nous a semblé qu'ils risquaient de fausser les résultats, et qu'ils méritaient une analyse propre, qui reste encore à mener. En conséquence, le nombre d'occurrences analysées s'en est trouvé réduit.

Ces difficultés nous ont amenées à conclure que l'étude sur corpus soulève certaines difficultés qui nécessitent de la compléter soit en aménageant les exemples, soit en retournant vers des exemples construits. Une autre voie de recherche à explorer pourrait reposer sur la recherche d'occurrences centrées sur le nom événementiel à tester et dont l'environnement correspond à l'application de tel ou tel test. Ce dernier point est cependant à mener avec précaution car, comme le prouve l'exemple (20) ci-dessus, le fait de rencontrer un nom événementiel avec avoir lieu par exemple ne garantit pas son interprétation processive. Enfin, et comme c'est très souvent le cas dans les études reposant sur des corpus, il s'avèrerait nécessaire de cumuler les indices et d'en dégager des convergences (réponses aux tests lorsqu'ils sont appliqués " manuellement » par le linguiste, réponses aux tests lorsqu'ils sont recherchés dans le corpus par l'entremise d'expressions régulières ou de fonctionnalités plus évoluées permettant d'accéder aux collocatifs de la cible, etc.). 
D. Beauseroy, E. Jacquey, M. L. KNitTEL

\section{Références bibliographiques}

Alexiadou A. (2001). Functional Structure in Nominals: Nominalization and Ergativity. Amsterdam and Philadelphia: John Benjamins.

Beauseroy D. (2009). Syntaxe et sémantique des noms abstraits statifs : des propriétés verbales et adjectivales aux propriétés nominales. Thèse de Doctorat, Nancy-Université / ATILF CNRS.

Beauseroy D. \& Knittel M.L. (2007). « Nombre et détermination: le cas des noms abstraits de qualité ", Rivista di Linguistica 19-2 : 231-262.

Borer H. (2005). Structuring sense (Vol. I: In name only). Oxford : Oxford University Press.

De Swart H. (1991). Adverbs of quantification. A generalized quantifier approach. Thèse, University de Groningen.

Godard D. (1986). "Les déterminants possessifs et les compléments de nom », Langue Française 72 : 102-122.

Grimshaw J. (1990). Argument Structure. Cambridge (Mass.) : MIT Press.

Haas P., Huyghe R. \& Marín R. (2008). « Du verbe au nom: calques et décalages aspectuels », in J. Durand, B. Habert \& B. Laks (éd.), Congrès Mondial de Linguistique Française. Paris : Institut de Linguistique Française, 20512065.

Heyd S. \& Knittel M.L. (2009). «Quelques remarques à propos des noms d'activités », Linguisticae Investigationes XXX, $1: 124-148$.

Kilgarriff M. R. \& Uí Dhonnchadha E. (2006). «Efficient corpus development for lexicography: building the New Corpus for Ireland », Language Resources and Evaluation Journal 40, 2 : 127-152.

Kleiber G. (2003). «Indéfini, Partitif et Adjectif : du nouveau. La lecture individualisante », Langages $151:$ 9-28.

Knittel M.L. (2008). «Les noms événementiels : des noms "à part"? », Communication présentée lors de la $1^{\text {re }}$ Journée 
d'études sur les Nominalisations (JENOM 1). Nancy, 1920 juin 2008.

Knittel M.L. (2010a). "Possession vs incorporation in the nominal domain; evidence from French event nominal dependencies », The Linguistic Review 27-2 : 177-230.

Knittel M.L. (2010b). "French Event nominals and pluralization », exposé présenté lors des $3^{\text {es }}$ Journées d'études sur les Nominalisations (JENOM 3). Paris, 17-18 juin 2010.

Kupferman L. (2004). Le mot de : domaines propositionnels et domaines quantificationnels. Bruxelles : De Boeck.

Kwon S. \& Zribi-Hertz A. (2004). « Number from a syntactic perspective : why plural marking looks 'truer' in French than in Korean », in O. Bonami \& P. Cabredo-Hofherr (eds), Empirical Issues in Syntax and Semantics 5. http://www.cssp.cnrs.fr/ eiss5/index_en.html.

Laca B. (2006). «Pluriactionnalité », in D. Godard, L. Roussarie \& F. Corblin (éd.), Sémanticlopédie: dictionnaire de sémantique, GDR Sémantique \& Modélisation, CNRS. http://www.semantique-gdr.net/dico/.

Melloni C. (2007). Polysemy in word formation: the case of deverbal nominals. Thèse de doctorat, Université de Vérone.

Milner J.-C. (1982). Ordres et raisons de langue. Paris : Seuil.

Mourelatos A. (1978). "Events, Processes and States", Linguistics and Philosophy 2 : 415-434.

Van de Velde D. (1995). Le spectre nominal: des noms de matière aux noms d'abstraction. Paris : Peeters.

Van Geenhoven V. (2004). "For-adverbials, frequentative aspect, and pluractionality », Natural Language Semantics $12: 135-190$.

Zribi-Hertz A. (1998). «Les syntagmes nominaux possessifs en français moderne : Syntaxe et morphologie », in J. Guéron \& A. Zribi-Hertz (éd.), La Grammaire de la Possession. Nanterre : Publidix, 129-166. 
D. BeAuseroy, E. JACQuey, M. L. KNITTEL

Annexes : Tests de distinction entre lecture événementielle et lecture résultative

\begin{tabular}{|c|c|c|}
\hline \multicolumn{3}{|c|}{ Tests aspectuels } \\
\hline & noms d'événements & exemples \\
\hline 1 & $\begin{array}{l}\text { [N1 de } \mathrm{N} 2] \text { sujet de se } \\
\text { dérouler }\end{array}$ & $\begin{array}{l}\text { la formation d'un gouvernement, qui } \\
\text { s'est déroulée hier } \\
\text { *la Confédération des Ecologistes, qui } \\
\text { s'est déroulée hier }\end{array}$ \\
\hline 2 & $\begin{array}{l}\text { [N1 de N2] sujet de } \\
\text { durer X temps }\end{array}$ & $\begin{array}{l}\text { l'accélération de la dégradation a duré } \\
\text { plusieurs mois } \\
\text { *les allées du pouvoir, qui ont duré } \\
\text { plusieurs mois }\end{array}$ \\
\hline 3 & $\begin{array}{l}\text { [N1 de N2] } \\
\text { complément de } \\
\text { effectuer / procéder à }\end{array}$ & $\begin{array}{l}\text { la signature d'une convention, } \underline{a} \\
\text { laquelle on procédera } \\
\text { La remise des prix, qu'on a effectuée } \\
\text { *l'ordre des Palmes, auquel on } \\
\text { procédera } \\
\text { *l'association du triathlon, qu'on } \\
\text { effectuera }\end{array}$ \\
\hline 4 & $\begin{array}{l}\text { [N1 de N2] sujet de } \\
\text { avoir lieu }\end{array}$ & $\begin{array}{l}\text { le traitement des déchets, qui a lieu à } \\
\text { l'usine } \\
\text { *l'intention des enfants, qui a lieu à } \\
\text { l'école }\end{array}$ \\
\hline 5 & $\begin{array}{l}\text { N2 éprouve / ressent } \\
\text { N1 }\end{array}$ & $\begin{array}{l}\text { la satisfaction du président } \\
\text { Le président ressent / éprouve de la } \\
\text { satisfaction } \\
\text { La satisfaction que le président ressent / } \\
\text { éprouve } \\
\text { *la convention éprouve / ressent la } \\
\text { signature }\end{array}$ \\
\hline 6 & $\begin{array}{l}\text { N1 dépend d'un verbe } \\
\text { support }\end{array}$ & $\begin{array}{l}\text { établir }+ \text { le classement (du concours) } \\
=>\text { classer }\end{array}$ \\
\hline 7 & $\begin{array}{l}\text { ajout possible de } \\
\text { circonstants temporels } \\
\text { de type en / pendant }+ \\
\text { durée. } \\
\text { [N1 de N2 en / } \\
\text { pendant }+ \text { durée] }\end{array}$ & $\begin{array}{l}\text { la formation d'un gouvernement en deux } \\
\text { jours } \\
\text { l'accélération de la dégradation } \\
\text { pendant plusieurs mois } \\
\text { *l'association du triathlon en / pendant } \\
\text { dix jours }\end{array}$ \\
\hline
\end{tabular}




\begin{tabular}{|c|c|c|}
\hline \multicolumn{3}{|c|}{ Tests argumentaux } \\
\hline 8 & $\begin{array}{l}\mathrm{N} 2 \text { renvoie à un } \\
\text { argument du verbe } \\
\text { correspondant à } \mathrm{N} 1\end{array}$ & $\begin{array}{l}\text { la prévention des maladies }=>\text { (cela) } \\
\text { prévient les maladies } \\
\text { la proposition du président }=>l e \\
\text { président propose } \\
\text { l'ordre des Palmes } \neq>\text { on ordonne les } \\
\text { Palmes / les palmes ordonnent }\end{array}$ \\
\hline $9 \mathrm{a}$ & $\begin{array}{l}\text { un complément } \\
\text { d'agent introduit par } \\
\text { par est possible } \\
\text { [ne s'applique que si } \\
8 \text { est positif et } 9 \mathrm{~b} \\
\text { négatif] }\end{array}$ & $\begin{array}{l}\text { le ramassage des ordures }+ \text { par les } \\
\underline{\text { éboueurs }} \\
\text { l'ordre du jour }+{ }^{*} \text { par le maire }\end{array}$ \\
\hline $9 b$ & $\begin{array}{l}\text { le complément } \\
\text { s'interprète comme le } \\
\text { sujet du verbe } \\
\text { correspondant à } \mathrm{N} 1 \\
\text { [ne s'applique que si } \\
8 \text { est positif et } 9 \mathrm{a} \\
\text { négatif] }\end{array}$ & $\begin{array}{l}\text { Le travail du cadre }=>\text { le cadre } \\
\text { travaille } \\
\text { l'entrée du village }=>* \text { le village entre }\end{array}$ \\
\hline 10 & $\begin{array}{l}\text { un complément } \\
\text { introduit par une autre } \\
\text { préposition est } \\
\text { possible ; } \\
\text { [V1 est transitif } \\
\text { indirect, et admet le } \\
\text { même complément } \\
\text { prépositionnel] }\end{array}$ & $\begin{array}{l}\text { Le départ du train pour Paris }[=>l e \\
\text { train part pour Paris] } \\
\text { La lutte de } Z \underline{\text { contre les parasites }[=>Z} \\
\text { lutte contre les parasites] }\end{array}$ \\
\hline
\end{tabular}

\begin{tabular}{|l|l|l|}
\hline \multicolumn{2}{|l|}{ Autres tests } \\
\hline \multicolumn{2}{|l|}{ Tests proposés par Grimshaw } \\
\hline 11 & $\begin{array}{l}\text { une subordonnée infinitive } \\
\text { exprimant le but est } \\
\text { possible ; }\end{array}$ & $\begin{array}{l}\text { le ramassage des ordures pour / } \\
\text { dans le but d'améliorer l'hygiène } \\
\text { *l'ordre du jour dans le but de } \\
\text { commencer la réunion }\end{array}$ \\
\hline
\end{tabular}


D. Beauseroy, E. Jacquey, M. L. Knittel

\begin{tabular}{|l|l|l|}
\hline 12 & $\begin{array}{l}\text { insertion possible } \\
\text { d'adjectifs de fréquence } \\
\text { (fréquent, occasionnel, etc.) } \\
\text { [l'acceptabilité de la } \\
\text { fréquence peut être liée à la } \\
\text { nécessité de pluraliser N1] }\end{array}$ & $\begin{array}{l}\text { la divagation des animaux } \\
=>\text { la divagation occasionnelle } \\
\text { des animaux } \\
\text { *le service fréquent des eaux }\end{array}$ \\
\hline \multicolumn{2}{|c|}{ Tests de distinction des noms résultatifs } \\
\hline 13 & $\begin{array}{l}\text { le nom déverbal est dans } \\
\text { une acception concrète, } \\
\text { matérielle }\end{array}$ & $\begin{array}{l}\text { le parking de la porte Désilles ; } \\
\text { l'entrée du village }\end{array}$ \\
\hline 14 & $\begin{array}{l}\text { le nom déverbal peut être } \\
\text { complément de se trouver, } \\
\text { se situer }\end{array}$ & $\begin{array}{l}\text { la scierie Virion se trouve / se } \\
\text { situe à l'entrée de la localité }\end{array}$ \\
\hline 15 & $\begin{array}{l}\text { être membre de N1 est } \\
\text { possible }\end{array}$ & $\begin{array}{l}\text { être membre de la Confédération } \\
\text { des Ecologistes }\end{array}$ \\
\hline Test de pluralisation & $\begin{array}{l}\text { nécessité de pluraliser N1 } \\
\text { avec un marqueur de } \\
\text { fréquence }\end{array}$ & \\
\hline 16 &
\end{tabular}

